\title{
Formulation And Evaluation Of Nanostructured Lipid Carriers (NLCs) Of 20(S)-Protopanaxadiol (PPD) By Box-Behnken Design
}

This article was published in the following Dove Press journal: International Journal of Nanomedicine

\author{
Min-Hwan Kim ' \\ Ki-Taek Kim ${ }^{2}$ \\ Seo-Yeon Sohn' \\ Jae-Young Lee ${ }^{3}$ \\ Chang Hyung Lee ${ }^{4}$ \\ Hee Yang ${ }^{4}$ \\ Bo Kyung Lee ${ }^{5}$ \\ Ki Won Lee ${ }^{4,5}$ \\ Dae-Duk Kim'

\begin{abstract}
'College of Pharmacy and Research Institute of Pharmaceutical Sciences, Seoul National University, Seoul, Republic of Korea; ${ }^{2}$ College of Pharmacy and Natural Medicine Research Institute, Mokpo National University, Jeonnam, Republic of Korea; ${ }^{3}$ College of Pharmacy, Chungnam National University, Daejeon, Republic of Korea; ${ }^{4}$ Department of Agricultural Biotechnology, Seoul National University, Seoul 08826 Republic of Korea; ${ }^{5}$ Research \& Development Center, BOBSNU Co., Ltd., Suwon, Gyeonggi-Do 16229, Republic of Korea
\end{abstract}

Background: 20(S)-Protopanaxadiol (PPD) has a higher anti-wrinkle effect than the other glycone forms of ginsenosides. However, as PPD has low solubility in water and a high molecular weight, it cannot easily penetrate the stratum corneum layer, which is the ratelimiting step of topical skin delivery. Thus, the objective was to enhance the topical skin deposition of PPD using an optimized nanostructured lipid carriers (NLC) formulation. NLC formulations were optimized using a Box-Behnken design.

Materials and methods: NLC formulations were optimized using a Box-Behnken design, where the amount of PDD $\left(\mathrm{X}_{1}\right)$, volume of the liquid lipid $\left(\mathrm{X}_{2}\right)$, and amount of surfactant $\left(\mathrm{X}_{3}\right)$ were set as the independent variables, while the particle size $\left(\mathrm{Y}_{1}\right)$, polydispersity index (PDI) $\left(\mathrm{Y}_{2}\right)$, and entrapment efficiency $(\mathrm{EE})\left(\mathrm{Y}_{3}\right)$ were dependent factors. An in vitro deposition study was performed using Strat- $\mathrm{M}^{\mathbb{R}}$ and human cadaver skin, while in vivo skin irritation effect of the NLC formulation was evaluated in humans.

Results: An NLC was successfully prepared based on the optimized formulation determined using the Box-Behnken design. The particle size, PDI, and EE of the NLC showed less than $5 \%$ difference from the predicted values. The in vitro deposition of PPD after the application of the NLC formulation on a Strat-M $\mathrm{M}^{\circledR}$ artificial membrane and human cadaver skin was significantly higher than that of the controls. Moreover, NLC formulations with and without PDD were not skin irritants in a human study.

Conclusion: An NLC formulation for the topical delivery of PPD was successfully optimized using the Box-Behnken design, and could be further developed to enhance the topical skin deposition of PPD.

Keywords: Box-Behnken design, 20(S)-Protopanaxadiol, nanostructured lipid carrier; NLC, Strat-MTM, topical delivery

\section{Introduction}

Of the two types of skin aging (i.e., intrinsic vs extrinsic aging), photo-aging is a typical extrinsic aging, which is mainly induced by ultraviolet (UV) light. ${ }^{1}$ The structure of the skin is changed by the activation of matrix metalloproteinases (MMPs) expressions caused by repeated exposure to UV. ${ }^{2}$ Among them, the expression of MMP-1 is known to increase the degradation of collagen in the dermis area, which results in many photoaging symptoms including wrinkles and skin roughness. Thus, inhibition of MMP-1 has been a key strategy for the prevention of photo-aging.

Ginsenoside is one of the principal active components that has been isolated from Panax ginseng, ${ }^{3}$ and is known to have many pharmacological effects
Correspondence: Dae-Duk Kim

College of Pharmacy and Research Institute of Pharmaceutical Sciences, Seoul National University, I Gwanak-ro, Gwanak-gu, Seoul 08826, Republic of Korea

Tel +8228807870

Fax +82 28739177

Email ddkim@snu.ac.kr 
including anti-cancer, anti- fatigue and skin-whitening effects. 20(S)-Protopanaxadiol (PPD) (Figure 1) is a deglycosylated metabolite of ginseng saponins, like compound $\mathrm{K}$. In addition to the various pharmacological potentials of PPD, including anti-cancer, anti-depressant and anti-inflammatory effects, ${ }^{4-6}$ it has also been reported that PPD has higher anti-wrinkle and skin-whitening effects than the other aglycone forms of ginsenosides in human skin equivalent models and human keratinocytes (HaCaTs) via inhibition of UV-induced MMP-1 expression. $^{7}$ Although the cosmeceutical potential of PPD is well established, studies of formulations for its topical delivery have been very limited. Since the aglycone form of ginsenoside, PPD is poorly soluble in water $(<50 \mathrm{ng} / \mathrm{mL})$. Moreover, a high molecular weight (460.7 Da) of PPD makes it not easy to penetrate the stratum corneum (SC) layer, which is the rate-limiting step of topical delivery. ${ }^{8,9}$ Thus, an optimized formulation is required in order to improve the topical skin deposition of PPD.

Among the many colloidal systems that have been studied for topical skin delivery, solid lipid nanoparticles (SLN) is one of the most promising carriers for lipophilic compounds. ${ }^{10}$ SLN is emulsion spheres with an average diameter of 10-1000 nm and have a "solid lipid" core stabilized by various surfactants. Cetyl palmitate is the most popular solid lipid for the preparation of SLNs, since it has an appropriately low melting point and has been approved by many regulatory agencies for topical application. ${ }^{11}$ SLN has various advantages as a topical delivery carrier, including controlled release, improved stability, and a skin hydration effect. ${ }^{12}$ However, solid lipids have a limited loading space, which leads to the expulsion of encapsulated molecules during storage. ${ }^{13}$ As such, nanostructured lipid carriers (NLC) was developed by mixing solid lipids with spatially incompatible oil (also

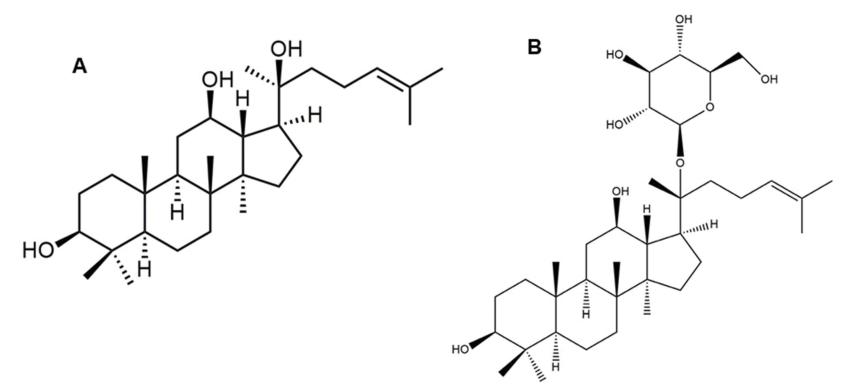

Figure I Chemical structure of (A) 20(S)-protopanaxadiol (PPD), an aglycone form of ginsenosides and (B) Compound $\mathrm{K}$. referred to as a "liquid lipid") as an improved generation of SLN. ${ }^{14,15}$ Because nanostructures lead to improved loading capacity and release properties, NLC formulations are used for many administration routes, including topical application. ${ }^{16}$ The topical application of prednicarbate in NLC formulation resulted in higher retention of the drug in the upper layer of the skin than that with an emulsionbased formulation. ${ }^{17}$ Thus, NLC formulations have been proposed to be suitable for cosmeceuticals that are intended to remain in the skin without permeation, thereby minimizing their systemic effects.

Recently, many statistical experimental designs have been used to more efficiently optimize formulations using fewer experiments, and to estimate the relative significance among variables. ${ }^{18}$ Several studies have reported that response surface methodologies (RSMs), which include central composite design (CCD), D-optimal design, and the Box-Behnken design (BBD), ${ }^{19}$ are useful for optimizing various formulations, including lipid particles, and for estimating the relationship between independent and dependent factors when interactions among the variables are complicated. ${ }^{20}$ BBD has a three-factor experimental design in which the three levels of the factors are located at the midpoints and the edges of the process space (Figure S1). As this cubic design has no vertices, it is easy to avoid experiments with extreme factors in BBD. Additionally, BBD requires fewer runs than any other three-level response surface design model. Therefore, $\mathrm{BBD}$ has been reported to be useful in the optimization of various nanocarriers with different applications. ${ }^{21}$

The objective of this study was to optimize an NLC formulation of PPD to enhance its topical skin deposition. After optimizing the PPD-loaded NLC formulation using $\mathrm{BBD}$, it was characterized by determining its mean particle size, encapsulation efficiency and morphology. Then, the in vitro deposition of PPD in Strat-MTM membranes and human cadaver skin was evaluated using LC-MS/MS analysis.

\section{Materials And Methods Materials}

20(S)-Protopanaxadiol (PPD) (purity $>98.0 \%$ ) was obtained from Xian Plant Bio-Engineering Co., Ltd. (Shaan Xi, China). PEG-8 Caprylic/Capric Glycerides (LAS), Labrafac $\mathrm{CC}$ and Lauoroglycol CC were obtained from Gattefosse Co. (Saint Priest, Cedex, France). Capmul MCM EP was obtained from ARITEC Co. (Columbus, OH, USA). Miglyol $812 \mathrm{~N}$ and Poloxamer 188 were gifts from OLEO Chemicals (Witten, 
Germany) and BASF Co. (Ludwigshafen, Germany), respectively. Limonene, Tween 20, Tween 80, polyethylene glycol 400 (PEG400) and isopropyl myristate were obtained from Sigma-Aldrich Co. (St. Louis, MO, USA). Cetyl palmitate was obtained from TCI (Tokyo Kasei, Japan). Strat-MTM was purchased from Merck Millipore (Billerica, MA, U.S.A.). HPLCgrade water, acetonitrile, and methanol were obtained from Thermo Fisher Scientific Co. (Pittsburgh, PA, USA).

\section{Selection Of The Liquid Lipid And Surfactant}

To determine the solubility of PPD in various liquid lipids and surfactants, an excessive amount of PPD (approximately 20 $\mathrm{mg}$ ) was added to a $2.0 \mathrm{~mL}$ tube containing $1 \mathrm{~mL}$ of each vehicle. The mixtures were shaken for $72 \mathrm{~h}$ at $25^{\circ} \mathrm{C}$ to reach an equilibrium state using a vortex shaker ( $50 \mathrm{rpm}$, Vortex-Genie 2; Scientific Industries, Inc., Bohemia, NY, USA). The mixtures were then centrifuged at $16,000 \mathrm{~g}$ for $5 \mathrm{~min}$, and the supernatant was passed through a $0.20 \mu \mathrm{m}$ syringe filter to remove the excess PPD. Finally, the PPD in the filtrates was determined using high performance liquid chromatographyultraviolet (HPLC-UV) analysis after appropriate dilution with methanol. Based on the solubility study of PPD (Table 1), Miglyol $812 \mathrm{~N}$ and Tween 20 were selected as the liquid lipid and surfactant, respectively, for the preparation of the NLC.

\section{Box-Behnken Experiment Design}

The Box-Behnken experimental design was used to statistically optimize the NLC formulation. The independent variables for optimization were the amount of PPD $\left(\mathrm{X}_{1}\right)$, the volume of the liquid lipid $\left(\mathrm{X}_{2}\right)$, and the amount of the surfactant $\left(\mathrm{X}_{3}\right)$, while the particle size $\left(\mathrm{Y}_{1}\right)$, polydispersity index $(\mathrm{PDI})\left(\mathrm{Y}_{2}\right)$ and entrapment efficiency $(\mathrm{EE})\left(\mathrm{Y}_{3}\right)$ were

Table I Solubility Of PPD In Various Liquid Lipids And Surfactants

\begin{tabular}{|l|l|l|}
\hline Vehicle & \multicolumn{2}{|l|}{ Solubility $(\mathrm{mg} / \mathrm{mL}) *$} \\
\hline Liquid lipid & Miglyol 8I2 N & $12.64 \pm 1.38$ \\
& Capmul MCM & $10.60 \pm 1.44$ \\
& L.A.S & $8.06 \pm 1.59$ \\
& Lauroglycol CC & $6.87 \pm 0.38$ \\
& Labrafac CC & $3.80 \pm 0.82$ \\
\hline \multirow{2}{*}{ Surfactant } & Tween 20 & $5.37 \pm 0.89$ \\
& Tween 80 & $3.55 \pm 0.64$ \\
& Isopropyl myristate & $2.53 \pm 0.73$ \\
& Limonene & $1.54 \pm 0.26$ \\
& PEG 400 & $0.02 \pm 0.01$ \\
\hline
\end{tabular}

Note: *Each value is the mean \pm SD $(n=3)$.

Abbreviation: L.A.S, PEG-8 Caprylic/Capric Glycerides. dependent factors. The Box-Behnken design sets points at the midpoint of each edge of a multidimensional cube and at the central point in triplicate (Figure S1). Thus, Table 2 shows the low $(-1)$, medium $(0)$ and high $(+1)$ levels of the independent variables, which were set based on the results of the preliminary experiments. Table 2 also shows the response criteria (i.e., the dependent variables, $\mathrm{Y}_{\mathrm{i}}$ ) for optimization of the NLC formulation. A design matrix with 15 experimental runs was constructed using the software Minitab $^{\circledR} 17$ (Minitab, Inc.) as shown in Table 3. The non-linear quadratic model generated by the design had the following form:

$$
\begin{aligned}
Y_{i}= & B_{0}+B_{1} X_{1}+B_{2} X_{2}+B_{3} X_{3}+B_{12} X_{1} X_{2}+B_{23} X_{2} X_{3} \\
& +B_{13} X_{1} X_{3}+B_{11} X_{1} X_{1}+B_{22} X_{2} X_{2}+B_{33} X_{3} X_{3}
\end{aligned}
$$

where $Y_{i}$ is the estimated response associated with each factor level combination, $\mathrm{B}_{0}$ is an intercept, and $\mathrm{B}_{1}$ to $\mathrm{B}_{33}$ are measured coefficients computed from the observed experimental values of $Y_{i}$. An obtained $P$-value less than 0.05 was considered statistically significant Analysis of variance (ANOVA) was also applied to determine the significance of the model.

\section{Preparation Of PPD-Loaded NLC}

Based on the 15 experimental runs generated by BBD (Table 3), the PPD-loaded NLCs were prepared using the method reported in the literature, with a slight modification. ${ }^{22}$ Briefly, the solid lipid (i.e., cetyl palmitate) was melted using a water bath at $65^{\circ} \mathrm{C}$, which is about $10^{\circ} \mathrm{C}$ higher than its melting point. Various amounts of PPD $\left(\mathrm{X}_{1}\right)$ and the liquid lipid (i.e., Miglyol $812 \mathrm{~N})\left(\mathrm{X}_{2}\right)$ were dissolved in a minimum amount of ethanol (less than $200 \mu \mathrm{L}$ ) and placed into the melted liquid

Table 2 Independent And Dependent Variables And Their Levels

\begin{tabular}{|c|c|c|c|}
\hline \multirow[t]{2}{*}{ Independent Variables } & \multicolumn{3}{|c|}{ Levels } \\
\hline & Low & Medium & High \\
\hline$X_{1}=\operatorname{PPD}(\%, w / v)$ & 0.05 & 0.1 & 0.15 \\
\hline$X_{2}=$ liquid lipid $(\%, v / v)$ & 0.5 & I & 1.5 \\
\hline$X_{3}=$ Tween $20(\%, v / v)$ & 0 & 1 & 2 \\
\hline Transformed values & -1 & 0 & +1 \\
\hline Dependent variables & \multicolumn{3}{|c|}{ Criteria: } \\
\hline $\mathrm{Y}_{1}=$ particle size $(\mathrm{nm})$ & \multicolumn{3}{|c|}{ Minimize } \\
\hline $\mathrm{Y}_{2}=$ polydispersity index & \multicolumn{3}{|c|}{ Close to 0.25} \\
\hline$Y_{3}=$ entrapment efficiency & \multicolumn{3}{|c|}{ Maximize } \\
\hline
\end{tabular}
In Box-Behnken Design

Abbreviation: PPD, 20(S)-protopanaxadiol. 
Table 3 Box-Behnken Design Matrix With Transformed Values And The Measured Response

\begin{tabular}{|c|c|c|c|c|c|c|}
\hline \multirow[t]{2}{*}{ Run } & \multicolumn{3}{|c|}{ Independent Variables } & \multicolumn{3}{|c|}{ Observed Data } \\
\hline & $\mathbf{x}_{1}$ & $\mathbf{X}_{2}$ & $\mathbf{X}_{3}$ & $\mathbf{Y}_{\mathbf{I}}$ & $\mathbf{Y}_{2}$ & $\mathbf{Y}_{3}$ \\
\hline 1 & -1 & 0 & -1 & $231.8 \pm 2.2$ & $0.179 \pm 0.037$ & $70.3 \pm 1.5$ \\
\hline 2 & 1 & -1 & 0 & $151.0 \pm 2.4$ & $0.290 \pm 0.008$ & $39.8 \pm 0.9$ \\
\hline 3 & 0 & 0 & 0 & $167.2 \pm 3.0$ & $0.250 \pm 0.007$ & $70.2 \pm 2.4$ \\
\hline 4 & 0 & 0 & 0 & $164.9 \pm 1.3$ & $0.257 \pm 0.001$ & $72.6 \pm 1.2$ \\
\hline 5 & I & 0 & I & $152.9 \pm 1.2$ & $0.290 \pm 0.009$ & $75.4 \pm 2.7$ \\
\hline 6 & 0 & 0 & 0 & $162.4 \pm 2.2$ & $0.252 \pm 0.022$ & $75.1 \pm 0.5$ \\
\hline 7 & 0 & I & -1 & $248.5 \pm 2.3$ & $0.220 \pm 0.030$ & $56.7 \pm 1.4$ \\
\hline 8 & 0 & I & 1 & $179.8 \pm 2.9$ & $0.250 \pm 0.013$ & $77.3 \pm 1.8$ \\
\hline 9 & -1 & I & 0 & $190.3 \pm 2.6$ & $0.250 \pm 0.004$ & $76.5 \pm 5.8$ \\
\hline 0 & -1 & 0 & 1 & $147.4 \pm 3.1$ & $0.272 \pm 0.012$ & $81.2 \pm 4.6$ \\
\hline II & 1 & 0 & -1 & $247.2 \pm 1.6$ & $0.249 \pm 0.010$ & $46.9 \pm 2.3$ \\
\hline 12 & 0 & -1 & -1 & $174.2 \pm 1.2$ & $0.262 \pm 0.010$ & $44.0 \pm 1.5$ \\
\hline 13 & -1 & -1 & 0 & $143.6 \pm 1.8$ & $0.280 \pm 0.001$ & $74.5 \pm 1.9$ \\
\hline 14 & 0 & -1 & 1 & $147.2 \pm 0.6$ & $0.298 \pm 0.013$ & $68.6 \pm 0.9$ \\
\hline 15 & I & 1 & 0 & $176.8 \pm 1.4$ & $0.250 \pm 0.013$ & $56.8 \pm 0.5$ \\
\hline
\end{tabular}

Abbreviations: $X_{1}$, percent of $20(S)$-protopanaxadiol; $X_{2}$, percent of liquid lipid; $X_{3}$, percent of Tween 20 ; $Y_{1}$, Particle size; $Y_{2}$, polydispersity index; $Y_{3}$, encapsulation efficiency.

lipid phase. Distilled water containing various amounts of Tween $20\left(\mathrm{X}_{3}\right)$ in $1 \%(\mathrm{v} / \mathrm{v})$ Poloxamer 188 were added to the lipid phase and ultrasonicated (VC-750; Sonics \& Materials, Inc., Newtown, CT, USA) with an amplitude of $26 \%$ and a pulse cycle of $2 \mathrm{~s}$ on and $3 \mathrm{~s}$ off. The mixture was then cooled to room temperature. Finally, the unentrapped PPD was removed by filtration (Wattman syringe filter; nylon; $0.45 \mu \mathrm{m}$ ).

\section{Characterization Of The NLC} Particle Size And Zeta Potential

The particle size $\left(\mathrm{Y}_{1}\right)$, polydispersity index $(\mathrm{PDI})\left(\mathrm{Y}_{2}\right)$ and zeta potential of the NLC were analyzed by an electrophoretic light-scattering (ELS) spectrophotometer (ELS 8000; Otsuka Electronics Co. Ltd., Tokyo, Japan). The samples were directly added in a quartz cuvette, and all measurements were carried out at $25^{\circ} \mathrm{C}$.

\section{Entrapment Efficiency}

The entrapment efficiency $\left(\mathrm{Y}_{3}\right)$ of the PPD in the NLC was calculated as described previously with slight modification. ${ }^{23}$ Briefly, after filtration to eliminate unentrapped PPD, $0.1 \mathrm{~mL}$ of the NLC formulation was put into $0.9 \mathrm{~mL}$ of methanol to completely disrupt the NLC. After centrifugation at $12,000 \mathrm{rpm}$ for $15 \mathrm{~min}$, the supernatant solution was analyzed using an HPLC system to determine the amount of PPD in the NLC. The entrapment efficiency was calculated using the following equation:

\author{
Entrapment efficiency $(\%)=$ \\ actual amount of PPD (mg) in NLC \\ total amount of PPD (mg) added in formulation $\times 100$
}

\section{Transmission Electron Microscopy (TEM) Analysis}

The morphology of the PPD-loaded NLC was viewed by a transmission electron microscopy (TEM) (LIBRA 120, Carl Zeiss, Germany) at $80 \mathrm{kV}$. A drop of PPD-NLC was loaded on a carbon-coated copper grid, and was negatively stained using $2 \%$ uranyl acetate and left to dry at room temperature.

\section{Power X-Ray Diffraction ( $\mathrm{PXRD}$ )}

To assess the crystallinity of the PPD and other excipients, pXRD patterns were obtained (BRUKER, German) using a $\mathrm{Cu}-\mathrm{K} \alpha$ source. Each sample was scanned in the $2 \theta$ range of 3 to $45^{\circ}$. NLC formulations with and without PPD were freeze-dried for pXRD analysis.

\section{In Vitro Deposition Of PPD Into The Strat-M ${ }^{\mathrm{TM}}$ Membrane}

The in vitro deposition of PPD into the Strat-M membrane after the application of the PPD-loaded NLCs to the membrane was investigated using a previously described method with slight modifications. ${ }^{24}$ Keshary-Chien diffusion cell with a surface area of $1.77 \mathrm{~cm}^{2}$ was used, and the receptor chamber $(13 \mathrm{~mL})$ was filled with $0.5 \%$ Tween 80 solution and was stirred continuously at $600 \mathrm{rpm}$ with a 
magnetic bar. The shiny side of the Strat-M membrane was placed on the donor part of the Keshary-Chien cell. An aliquot $(1.0 \mathrm{~mL})$ of an NLC solution or a lipid solution (i.e., Miglyol $812 \mathrm{~N}$ ) containing $0.05 \%(\mathrm{w} / \mathrm{v})$ PPD was filled into the donor compartment. PPD in an aqueous suspension containing $5 \%(\mathrm{w} / \mathrm{w})$ propylene glycol was also evaluated for comparison. The donor compartment was covered with parafilm to avoid evaporation. The Strat-M membrane was removed from the diffusion cells $3 \mathrm{~h}$ or $6 \mathrm{~h}$ after the sample was added into the donor compartment and washed three times with methanol. The membranes were placed into a tube containing $1.5 \mathrm{~mL}$ of acetone and methanol $(7: 3, \mathrm{v} / \mathrm{v})$, which was then shaken for $3 \mathrm{hr}$ by using vortex shaker (Vortex-Genie 2; Scientific Industries, Inc., Bohemia, NY, USA) to extract PPD. After centrifugation for $5 \mathrm{~min}$ at $16,000 \mathrm{~g}$, an aliquot $(1.0 \mathrm{~mL})$ of the supernatant was evaporated using a nitrogen gas stream. The dried aliquot was reconstituted with $0.5 \mathrm{~mL}$ of methanol, and the concentration of PPD was analyzed using LC-MS/MS.

\section{In Vitro Deposition Of PPD Into Human Cadaver Skin}

The in vitro deposition of PPD into human cadaver skin after the application of the optimized formulation of the PPD-loaded NLC to the skin was investigated using the same diffusion cells with a slight modification. ${ }^{8}$ Male human cadaver skin from the thigh was obtained from HansBiomed (Seoul, Korea), and was stored at $-20{ }^{\circ} \mathrm{C}$. The "Enforcement Rule of the Bioethics and Safety Act (article 33)" in Korea states that studies using research materials separated and processed from human body to be used by the general public are not required approval from institutional review board (IRB) or ethics committee, in the case where human body materials are not directed collected. $^{25}$ The human cadaver skin was removed from the diffusion cells at $3 \mathrm{~h}$ or $6 \mathrm{~h}$ after applying the NLC formulation and washed with methanol. Then, the epidermis and dermis were separated by placing the skin in $60^{\circ}$ $\mathrm{C}$ distilled water for $2 \mathrm{~min}$, after which the epidermis was peeled off. The epidermis and dermis parts were separately cut into small pieces and ground into a powder using liquid nitrogen. They were then collected in a tube and extracted with methanol by shaking for $3 \mathrm{~h}$. After centrifugation for $5 \mathrm{~min}$ at $16,000 \mathrm{~g}$, the amount of PPD in the supernatant was analyzed using LC-MS/MS.

\section{HPLC Analysis Of PPD}

An HPLC-UV system equipped with a pump (Waters 1515; Waters Company, Milford, Massachusetts), a UV/ Vis detector (Waters 2487), and an automatic injector (Waters 717 plus) was used to determine the EE of PPD in the NLC formulations. The samples were injected into a reverse-phase $\mathrm{C} 18$ column (Kinetex $\mathrm{C} 18 ; 250 \times 4.6 \mathrm{~mm} 2,5$ $\mu \mathrm{m}$; Phenomenex). The mobile phase consisted of acetonitrile and water $(95: 5, \mathrm{v} / \mathrm{v})$ at a flow rate of $1.0 \mathrm{~mL} / \mathrm{min}$. The detection wavelength and injection volume were 210 nm and $20 \mu \mathrm{L}$, respectively.

The amount of PPD deposited in the Strat-MTM membrane and human cadaver skin was determined using a liquid chromatography-mass spectrometry/mass spectrometry (LC-MS/MS) system (Agilent Technologies, Santa Clara, CA, USA). The Agilent Technologies 1260 Infinity HPLC system was equipped with an Agilent Technologies 6430 Triple Quad LC-MS system and a Hypersil BDS C18 column $(50 \mathrm{~mm} \times 4.6 \mathrm{~mm} \times 5 \mathrm{~mm}$; Thermo Fisher Scientific Co.). ${ }^{24}$ The mobile phase consisted of $93 \%$ acetonitrile and $7 \%$ water containing $0.2 \%$ formic acid $(\mathrm{v} / \mathrm{v})$ at a flow rate of $0.37 \mathrm{~mL} / \mathrm{min}$. The injection volume was $10 \mu \mathrm{L}$. PPD was determined in the multiple reaction-monitoring (MRM) mode with positive electrospray ionization (ESI). The gas flow, gas temperature, nebulizer pressure, and capillary voltage were $9 \mathrm{~L} / \mathrm{min}, 120^{\circ} \mathrm{C}, 25 \mathrm{psi}$, and $6,000 \mathrm{~V}$, respectively. The $\mathrm{m} / \mathrm{z}$ values of the precursor to the product ion were $\mathrm{m} / \mathrm{z} 461.4 \rightarrow \mathrm{m} / \mathrm{z} 425.5$ for PPD analysis. The collision energy, fragment voltage, and cell accelerator voltage and were $4 \mathrm{eV}, 111 \mathrm{~V}$, and $1 \mathrm{~V}$, respectively. The retention time of PPD was $1.20 \mathrm{~min}$. All LC-MS/MS data were processed using MassHunter Workstation Software Quantitative Analysis (vB.05.00; Agilent Technologies). The standard samples for the calibration curve were serially diluted with methanol from a stock solution $(1 \mathrm{mg} / \mathrm{mL})$. The response of the detector was linear in the concentration range $2-1000 \mathrm{ng} / \mathrm{mL}$, and the mean correlation coefficient $\left(\mathrm{r}^{2}\right)$ for the calibration curve was higher than 0.999 . The lower limit of quantification (LLOQ) was $2 \mathrm{ng} / \mathrm{mL}$, and there was no interference from any other substance.

\section{In Vivo Human Skin Irritation Study}

The human skin irritation effect of the PPD-loaded NLC formulation was evaluated at the Korea Dermatology Research Institute (Seongnam, Korea) in 32 healthy Korean female volunteers (age 21-58). Before applying 
the samples, the back of each subject was cleaned with $70 \%$ ethanol. Then, $15 \mu \mathrm{L}$ of each sample (ie, NLC with or without PPD) was placed in a Finn chamber on the scapular region of each subject's back, and then fixed with plaster for $24 \mathrm{hrs}$. The skin irritation scores were measured at $30 \mathrm{~min}, 24 \mathrm{hrs}$, and $48 \mathrm{hrs}$, based on the criteria in Table S1. The average irritation score of each subject was calculated using the following equation:

Irritation score $=\frac{\sum \text { irritation score at } 30 \mathrm{~min}, 24 \text { and } 72 \mathrm{hr}}{\text { total number of obervations }}$

The average irritation score of all human subjects was then calculated in order to determine the irritation effect of the NLC formulations with and without PPD based on the criteria in Table S2. The study protocol was approved by the institutional review board (IRB) (KDRI-IRB-16,977). All volunteers were provided written informed consent of the study and the protocol was approved by the Korea Dermatology Research Institute IRB, and followed the principles of the Declaration of Helsinki. The irritation scores were calculated based on a slight modification of the criteria of International Contact Dermatitis Research Group (ICDRG) and Personal Care Products Council (PCPC) safety evaluation guidelines.

\section{Statistical Analysis}

All experiments were carried out at least three times, and the data were presented as the mean \pm standard deviation (SD). Statistical analyses were performed using the twotailed $t$-test or analysis of variance (ANOVA) with post-hoc test (IBM SPSS Statistics software, version 21.0; IBM Corp, Armonk, NY, USA), and $\mathrm{p}<0.05$ indicated a statistically significant difference.

\section{Results}

\section{Solubility Study}

As PPD is poorly soluble in water $(<50 \mathrm{ng} / \mathrm{mL})$, the selection of a suitable vehicle was one of the most important process for the formulation of the NLCs. Among the liquid lipids tested, PPD showed the highest solubility in Miglyol $812 \mathrm{~N}(12.64 \pm 1.39 \mathrm{mg} / \mathrm{mL})$, followed by Capmul MCM, L.A.S, Lauroglycol CC and Labrafac CC. Tween 20 showed the highest solubility among surfactants of $5.37 \pm 0.89 \mathrm{mg} / \mathrm{mL}$, followed by Tween 80 , isopropyl myristate, limonene and PEG 400. (Table 1). Thus, Miglyol $812 \mathrm{~N}$ and Tween 20 were selected for the optimization of the formulation with the solid lipid (i.e., cetyl palmitate) for the preparation of the NLCs.

\section{Box-Behnken Design Of Experiment And Data Analysis}

Table 3 shows the 15 experimental runs carried out for the optimization of NLC formulation, together with all the responses (i.e., dependent variables) observed. The responses $\left(\mathrm{Y}_{1}, \mathrm{Y}_{2}\right.$, and $\left.\mathrm{Y}_{3}\right)$ were fitted to the multiple regression analysis to give the following quadratic polynomial equations (Table 4):

$$
\begin{aligned}
\mathrm{Y}_{1}= & 138.0+296 \mathrm{X}_{1}+95.5 \mathrm{X}_{2}-80.7 \mathrm{X}_{3}-209 \mathrm{X}_{1} \mathrm{X}_{2} \\
& -50 \mathrm{X}_{1} \mathrm{X}_{3}-14.9 \mathrm{X}_{2} \mathrm{X}_{2}+25.7 \mathrm{X}_{3} \mathrm{X}_{3}
\end{aligned}
$$

$$
\begin{aligned}
& \mathrm{Y}_{2}=0.261+0.647 \mathrm{X}_{1}-0.160 \mathrm{X}_{2}+0.060 \mathrm{X}_{3}-0.130 \mathrm{X}_{1} \mathrm{X}_{2} \\
& -0.270 \mathrm{X}_{1} \mathrm{X}_{3}+0.068 \mathrm{X}_{2} \mathrm{X}_{2}-0.002 \mathrm{X}_{3} \mathrm{X}_{3} \\
& \mathrm{Y}_{3}=68.8-447 \mathrm{X}_{1}+41.4 \mathrm{X}_{2}+0.30 \mathrm{X}_{3}+150 \mathrm{X}_{1} \mathrm{X}_{2} \\
& +87.8 \mathrm{X}_{1} \mathrm{X}_{3}-23.1 \mathrm{X}_{2} \mathrm{X}_{2}+0.76 \mathrm{X}_{3} \mathrm{X}_{3}
\end{aligned}
$$

The particle size of the NLC varied from 143.6 to $248.5 \mathrm{~nm}$ (Table 3). The quadratic equation indicated that $\mathrm{Y}_{1}$ (particle

Table 4 Results Of Regression Analysis

\begin{tabular}{|l|l|l|l|l|l|l|}
\hline \multirow{2}{*}{ Parameter } & \multicolumn{2}{l|}{ Particle Size $\left(\mathbf{Y}_{\mathbf{1}}\right)$} & \multicolumn{2}{l|}{ PDI $\left(\mathbf{Y}_{2}\right)$} & \multicolumn{2}{l|}{ EE $\left(\mathbf{Y}_{\mathbf{3}}\right)$} \\
\cline { 2 - 7 } & Coefficient & p-value & Coefficient & p-value & \multicolumn{2}{l|}{ Coefficient } \\
\hline Intercept & 138 & 0.003 & 0.261 & 0.000 & 68.8 & p-value \\
$X_{1}$ & 296 & 0.739 & 0.647 & 0.078 & -447 & 0.000 \\
$X_{2}$ & 95.5 & 0.004 & -0.160 & 0.019 & 41.4 & 0.001 \\
$X_{3}$ & -80.7 & 0.000 & 0.060 & 0.005 & 0.30 & 0.044 \\
$X_{1} X_{2}$ & -209 & 0.513 & -0.130 & 0.713 & 150 & 0.001 \\
$X_{1} X_{3}$ & -50 & 0.752 & -0.270 & 0.155 & 87.8 & 0.239 \\
$X_{2} X_{2}$ & -14.9 & 0.650 & 0.068 & 0.093 & -23.1 & 0.177 \\
$X_{3} X_{3}$ & 25.7 & 0.014 & -0.002 & 0.814 & 0.76 & 0.098 \\
$R^{2}$ & 0.910 & & 0.841 & & 0.904 & 0.809 \\
\hline
\end{tabular}

Abbreviations: PDI, polydispersity index; $\mathrm{EE}$, encapsulation efficiency; $\mathrm{X}_{1}$, percent of 20(S)-protopanaxadiol; $\mathrm{X}_{2}$, percent of liquid lipid; $\mathrm{X}_{3}$, percent of Tween 20; $R^{2}$, R-squared. 
size) was significantly influenced by $\mathrm{X}_{2}$ (volume of liquid lipid) and $X_{3}$ (amount of Tween 20), while the effect of $X_{1}$ (amount of PPD) was not statistically significant (Table 4). Out of the three independent variables, $\mathrm{X}_{3}$ showed the most prominent negative effect on the particle size of the NLC. The observed PDI values $\left(\mathrm{Y}_{2}\right)$ varied from 0.179 to 0.298 (Table 3). Although the two independent variables $\left(\mathrm{X}_{2}\right.$ and $\mathrm{X}_{3}$ ) showed statistically significant influences on the PDI $(p<0.05)$, the positive effect of $X_{3}$ was the most noticeable (Table 4). The observed EE values $\left(\mathrm{Y}_{3}\right)$ varied from 39.8 to $81.2 \%$ (Table 3 ). Although the influence of all three variables on the EE was statistically significant ( $p<0.01), \mathrm{X}_{1}$ and $X_{3}$ had a more obvious negative and positive influences, respectively, than $\mathrm{X}_{2}$ (Table 4). Moreover, the 2D contour plots and 3D response surface plots in Figure 2 also consistently demonstrated these relationships among the independent and dependent variables.

\section{Validation Of Optimized NLC Formulation}

Based on the optimization plots, the optimal values of $\mathrm{X}_{1}, \mathrm{X}_{2}$, and $\mathrm{X}_{3}$ were $0.05 \%(\mathrm{w} / \mathrm{v})$ PPD, $0.80 \%(\mathrm{v} / \mathrm{v})$ liquid lipid, and $1.17 \%(\mathrm{v} / \mathrm{v})$ Tween 20, respectively (Figure 3). The predicted values of the responses $\mathrm{Y}_{1}$ (particle size), $\mathrm{Y}_{2}$ (PDI), and $\mathrm{Y}_{3}$ (EE) were $149.3 \mathrm{~nm}, 0.250$, and $77.3 \%$, respectively. It is known that there is high possibility to obtain optimum responses, if the total and individual desirability values are close to $1 .{ }^{26}$ Table 5 shows the experimentally observed $\mathrm{Y}_{1}$, $\mathrm{Y}_{2}$, and $\mathrm{Y}_{3}$ to be $148.7( \pm 1.5) \mathrm{nm}, 0.250( \pm 0.014)$, and 78.2 $( \pm 3.1) \%$, respectively. These corresponded to relative percentages of $99.6 \%, 100 \%$, and $101.2 \%$ for $\mathrm{Y}_{1}, \mathrm{Y}_{2}$, and $\mathrm{Y}_{3}$, respectively, confirming that the Box-Behnken design model successfully optimized the PPD-loaded NLC formulation. The zeta potential of the optimized NLC formulation was $-11.5 \pm 1.6 \mathrm{mV}$, which showed that NLC particles were stable based on previous results. ${ }^{24}$

\section{TEM Analysis And X-Ray Diffraction Studies Of The Optimized NLC}

The morphology of the optimized NLC formulation was observed using TEM. The TEM image in Figure 4 shows the circle or oval shape of the particles; no aggregation of the particles was observed. The diameter of the NLCs observed in the TEM images (about $150 \mathrm{~nm}$ ) was in good agreement with data obtained using an electrophoretic light-scattering spectrophotometer.

Figure 5 shows the pXRD data of PPD, cetyl palmitate, poloxamer, and a physical mixture of the three, together with that of the NLC formulation with and without PPD. Although the crystalline peaks of PPD were clearly observed for the physical PPD/cetyl palmitate/poloxamer mixture, they were not observed for the NLC formulations, indicating that the PPD encapsulated in NLCs had an amorphous form.

\section{In Vitro Deposition Of PPD Into Strat-MTM Membrane And Human Cadaver Skin}

Figure 6 shows the amount of PPD deposited into the Strat-M membranes in the in vitro experiments $3 \mathrm{~h}$ and $6 \mathrm{~h}$ after the application of the aqueous suspension, lipid solution, or NLC formulation. The amounts of deposited PPD followed the order NLC formulation $>$ lipid solution $>$ suspension. It is
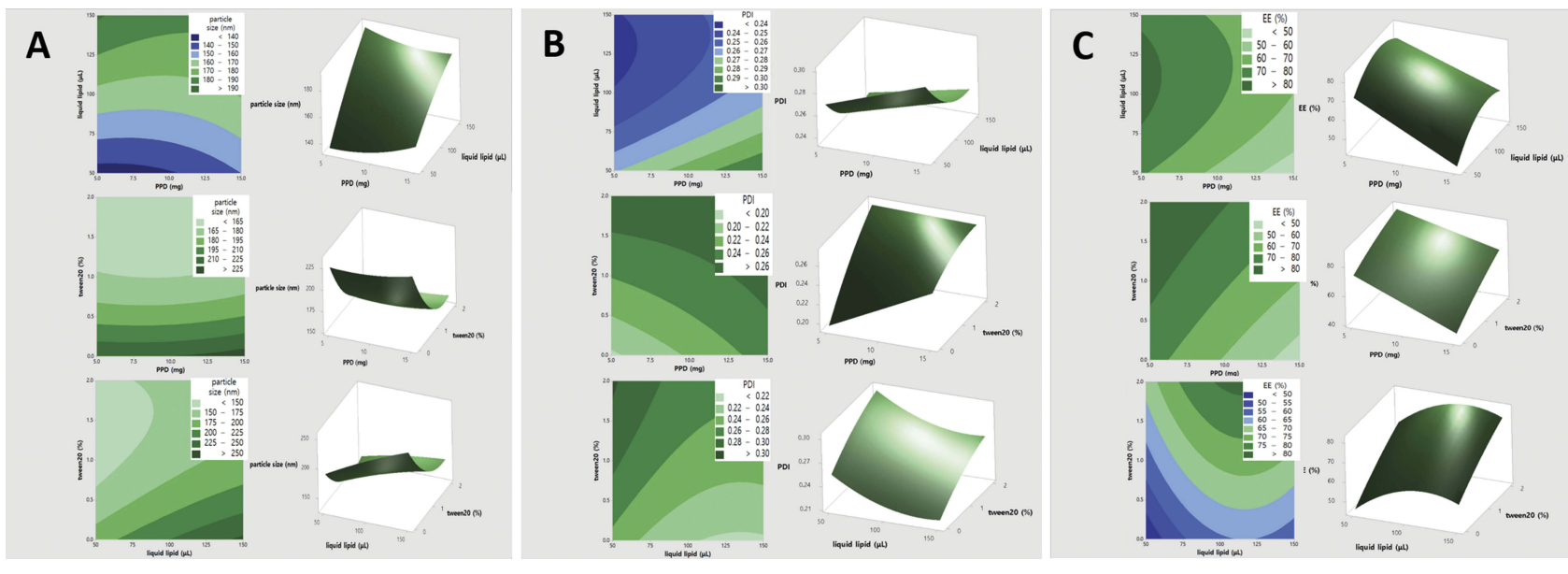

Figure 2 2D contour plots and 3D response surface plots for the effect of PPD amount, volume of oil, and surfactant amount on (A) particle size, (B) polydispersity index $(\mathrm{PDI})$, and $(\mathbf{C})$ entrapment efficiency $(\mathrm{EE})$. 


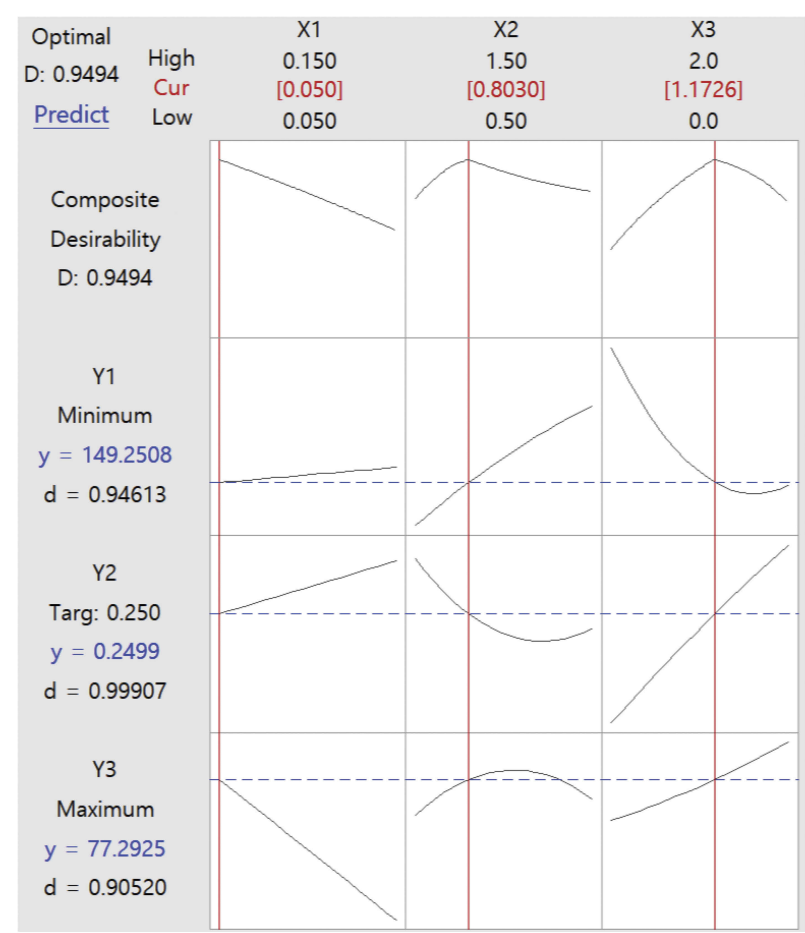

Figure 3 Optimization plots of $Y_{1}, Y_{2}$ and $Y_{3}$ versus $X_{1}, X_{2}$ and $X_{3}$.

notable that the deposition of PPD into the Strat-M membrane from the lipid solution (i.e., Miglyol $812 \mathrm{~N}$ ) at $3 \mathrm{~h}$ and $6 \mathrm{~h}$ was about 155 times and 57 times higher $(\mathrm{p}<0.001)$ than the amount deposited from the suspension respectively. Moreover, when Miglyol 812N was used as the liquid lipid in the NLCs, the deposition of PPD at $3 \mathrm{~h}$ and $6 \mathrm{~h}$ was about 3 times and 1.8 times enhanced, respectively $(\mathrm{p}<0.001)$.

Figure 7 shows the in vitro deposition of PPD in the epidermis and dermis layers of the human cadaver skin $3 \mathrm{~h}$ and $6 \mathrm{~h}$ after applying the liquid solution or NLC formulation of PPD. Figure 7A shows that the deposition amount of PPD in

Table 5 Comparison Between The Predicted Yi Values Obtained By BBD And Their Actual Measurement Values Of The Optimized PPD-Loaded NLC Formulation

\begin{tabular}{|l|l|l|l|}
\hline Response & $\begin{array}{l}\text { Predicted } \\
\text { Value (A) }\end{array}$ & $\begin{array}{l}\text { Experimental } \\
\text { Value (B) }\end{array}$ & $\begin{array}{l}\text { Relative } \\
\text { Percentage } \\
(100 \times \text { B/A) } \\
(\%)\end{array}$ \\
\hline $\begin{array}{l}\text { Particle size } \\
\left(\mathrm{nm} ; \mathrm{Y}_{1}\right)\end{array}$ & 149.2 & $148.7 \pm 1.5$ & 99.6 \\
$\begin{array}{l}\mathrm{PDI}\left(\mathrm{Y}_{2}\right) \\
\mathrm{EE}\left(\% ; \mathrm{Y}_{3}\right)\end{array}$ & 0.250 & $\begin{array}{l}0.250 \pm 0.014 \\
78.2 \pm 3.1\end{array}$ & $\begin{array}{l}100.0 \\
101.2\end{array}$ \\
\hline
\end{tabular}

Note: Data were expressed as mean \pm standard deviation $(n=3)$.

Abbreviations: BBD, Box-Behnken design; PPD, 20(S)-protopanaxadiol; NLC, nanostructured lipid carrier; PDI, polydispersity index; EE, encapsulation efficiency.

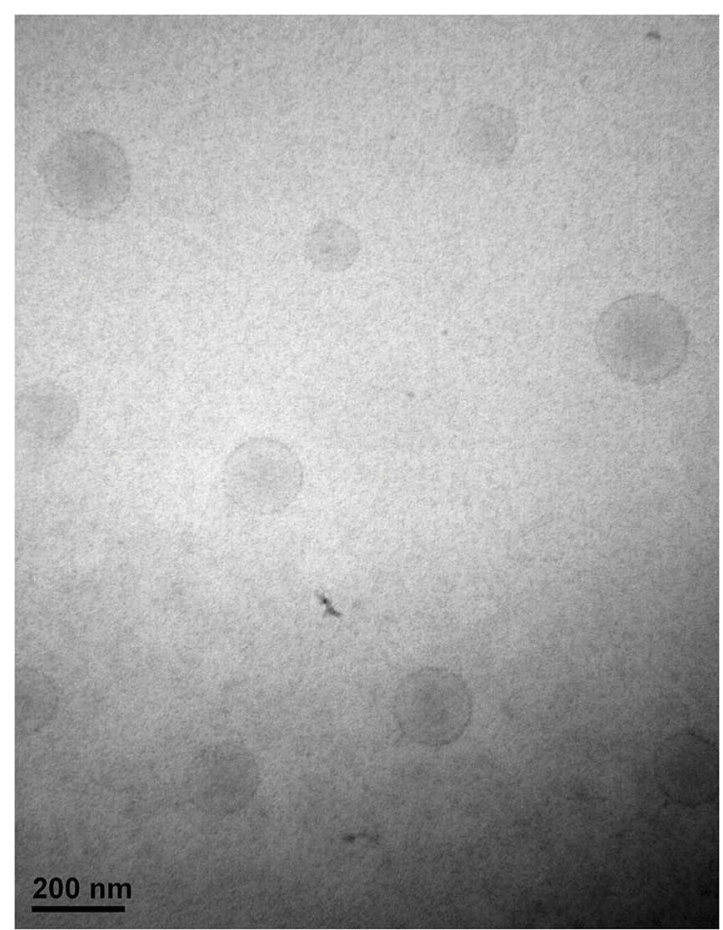

Figure 4 TEM image of NLC optimized by Box-Behnken design. The scale bars represent $200 \mathrm{~nm}$.
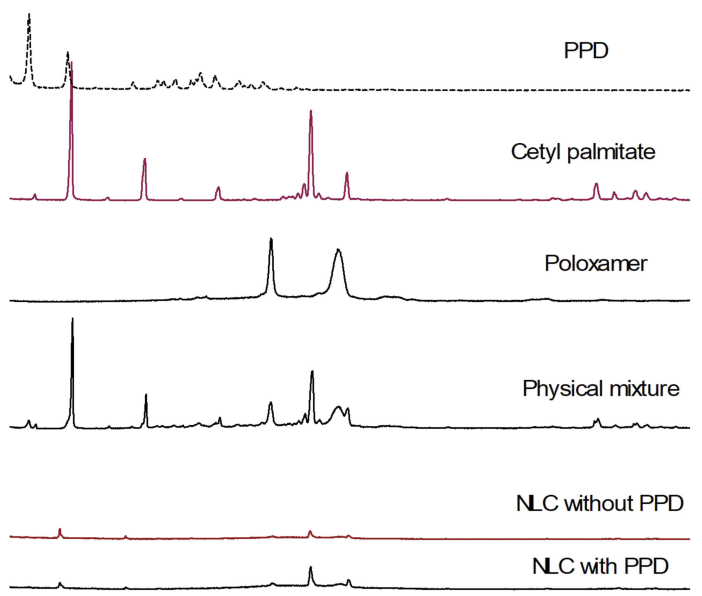

$2 \theta$

Figure 5 X-ray diffraction patterns of PPD, cetyl palmitate, poloxamer and physical mixture of PPD/cetyl palmitate/poloxamer, freeze-dried NLC without PPD, and freeze-dried NLC with PPD.

the epidermis layer from the NLC formulation was 4.5 times and 4.8 times higher at both $3 \mathrm{~h}$ and $6 \mathrm{~h}$ than that from the lipid solution. However, as shown in Figure 7B, the amount of PPD deposited in the dermis layer from the lipid solution was not 


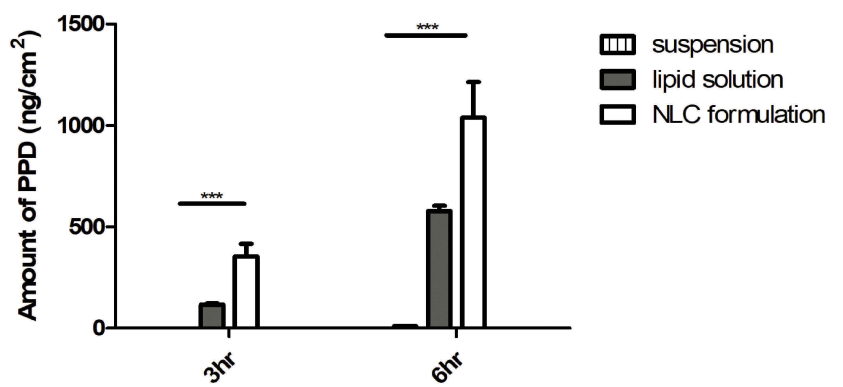

Figure 6 In vitro deposition amount of PPD in the Strat-M ${ }^{\text {TM }}$ membrane at $3 \mathrm{hr}$ and $6 \mathrm{hr}$ after application of $1.0 \mathrm{~mL}$ of aqueous solution, lipid solution or optimized NLC formulation containing $0.5 \%(w / v)$ of PPD. Data were expressed as the mean \pm standard deviation $(n=4)$. ${ }^{* * *} p<0.0001$.

significantly enhanced, while the amount deposited from the NLC formulation was significantly increased and was higher than the amount deposited from the lipid solution at $6 \mathrm{~h}$. These results indicated that the NLC formulation enhanced the in vitro human skin deposition of PPD in the epidermis layer, as well as significantly increasing its permeation through the epidermis layer, thereby significantly enhancing the deposition of PPD in the dermis layer. Thus, the total deposition amount of PPD in the skin was also significantly increased $6 \mathrm{~h}$ after the application of the NLC formulation and was greater than that from the lipid solution (Figure 7C).

\section{In Vivo Human Skin Irritation Study In Human}

Table 6 summarizes the results of the in vivo human skin irritation study after a single dose application of the NLC formulations with and without PPD. The average irritation score of all human subjects was nearly zero, confirming that the NLC formulations with and without PPD did not cause irritation in human skin, based on the criteria in Table S2.

\section{Discussion}

Prior to the optimization of the NLC formulation of PPD using the statistical experimental design, various liquid lipids and surfactants that were biocompatible, safe and commonly used for skin applications were screened in the solubility study. Since PPD showed the highest solubility in Miglyol $812 \mathrm{~N}$ and Tween 20 (Table 1), these were chosen as the liquid lipid and surfactant, respectively. Then, the NLC formulation was optimized using cetyl palmitate as the solid lipid, since it is one of the most common solid lipid cores in the preparation of SLN due to its suitable melting point and low toxicity. ${ }^{11}$ Moreover, $1 \%$ (v/v) Poloxamer 188 was added during the emulsifying
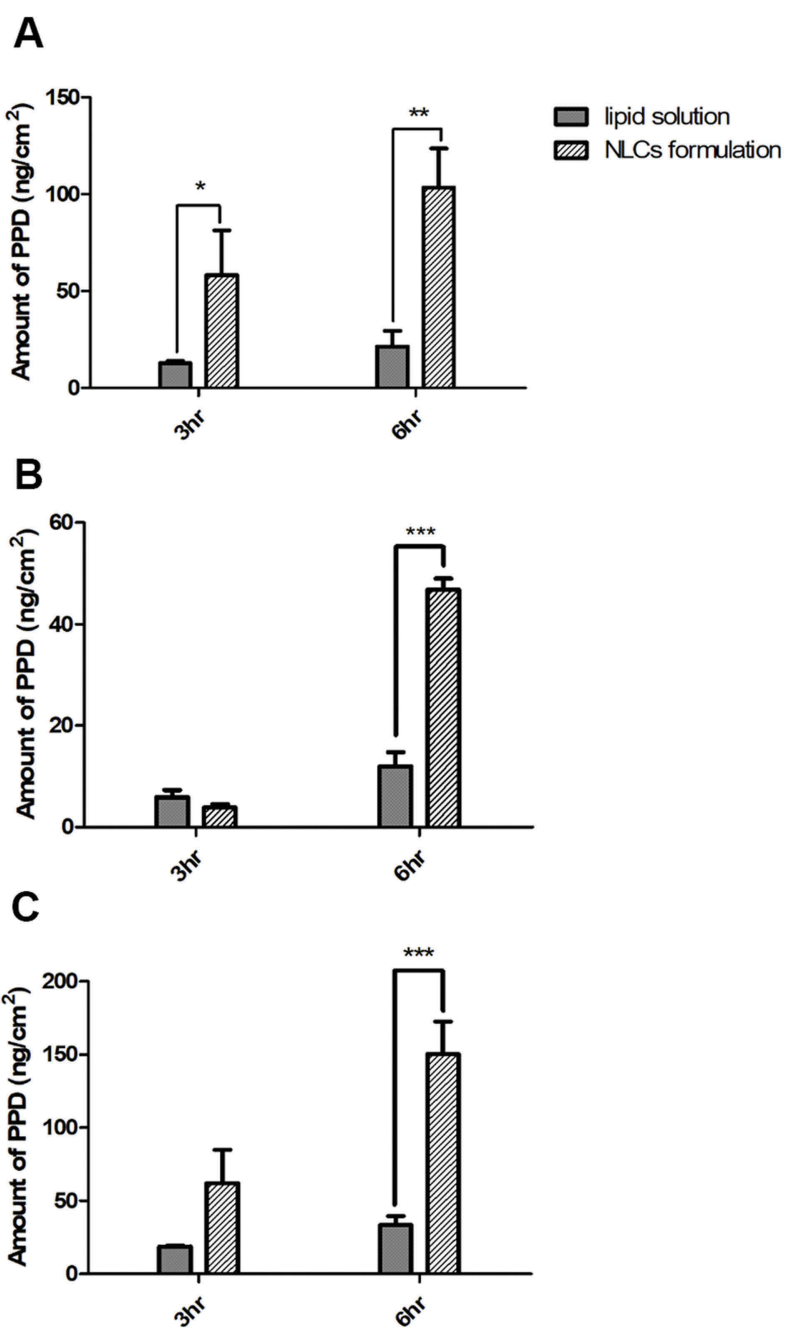

Figure 7 In vitro deposition amount of PPD in the epidermis (A), dermis (B), and total skin (C) of the human cadaver skin at $3 \mathrm{hr}$ and $6 \mathrm{hr}$ after application of $1.0 \mathrm{~mL}$ of liquid solution or optimized NLC formulation containing $0.5 \%(\mathrm{w} / \mathrm{v})$ of PPD. Data were expressed as the mean \pm standard deviation $(n=3)$. ${ }^{*}<0.05$; $*^{*} \mathrm{p}<0.01$; *** $\mathrm{p}<0.001$.

process to increase the physical stability of the NLC formulations. ${ }^{27}$ In our preliminary study, the addition of up to $0.5 \%(\mathrm{v} / \mathrm{v})$ Poloxamer 188 significantly decreased the particle size of cetyl palmitate SLN (Table S3) due to its steric stabilization effect.

In order to validate the Box-Behnken design model, NLC was prepared using the optimized formulation, and the experimentally measured response values were compared with the predicted ones. As shown in the results in Table 5, the experimental values of $Y_{1}, Y_{2}$, and $Y_{3}$ from the optimized NLC formulation were very close to the predicted response values. These results confirmed that the BBD model successfully optimized the PPD-loaded NLC formulation. Statistical experimental designs are 
Table 6 Average Irritation Score Of All Human Subjects $(n=32)$ After Single Dose Application Of The Optimized NLC Formulations With Or Without PPD

\begin{tabular}{|l|l|l|}
\hline \multirow{2}{*}{} & \multicolumn{2}{|l|}{$\begin{array}{l}\text { Optimized NLC } \\
\text { Formulation }\end{array}$} \\
\cline { 2 - 3 } & Without PPD & With PPD \\
\hline $\begin{array}{l}\text { Average human skin irritation score } \\
\text { Irritation criteria }\end{array}$ & $\begin{array}{l}0.00 \\
\text { No irritancy }\end{array}$ & $\begin{array}{l}0.00 \\
\text { No irritancy }\end{array}$ \\
\hline
\end{tabular}

Abbreviation: PPD, 20(S)-protopanaxadiol.

also useful to estimate the relative significance of variables. For example, the quadratic equation for $\mathrm{Y}_{1}$ (particle size) showed that the amount of Tween $20\left(\mathrm{X}_{3}\right)$ had the most significant negative effect (Table 4). The 2D-contour plots and 3D-response surface plots in Figure 2A also consistently showed that the particle size of the NLC decreased as the amount of Tween 20 increased. It has been reported that increasing the surfactant content can reduce the interfacial tension of the surface of the particles, thereby reducing the particle size of the emulsion. ${ }^{28}$ Moreover, Tween $20\left(\mathrm{X}_{3}\right)$ also showed a significant positive influence on the $\mathrm{EE}\left(\mathrm{Y}_{3}\right)$, as did the liquid lipid $\left(\mathrm{X}_{2}\right)$, as shown in Table 4 and Figure $2 \mathrm{C}$. Increasing the amount of liquid lipid $\left(\mathrm{X}_{2}\right)$ and surfactant $\left(\mathrm{X}_{3}\right)$ could increase the solubility of PPD in the inner matrix space due to the imperfections of the inner phase of the NLC formulation. ${ }^{29}$

Strat-M $\mathrm{M}^{\mathrm{TM}}$ is a commercially available artificial membrane that mimics human skin. It is composed of three layers of polyester sulfone with different compositions and structures. ${ }^{30}$ Many researchers have reported that Strat-M is one of the alternative membranes for estimating skin permeation and deposition. ${ }^{31}$ We previously reported a good relationship between the in vitro deposition profiles of PPD into Strat-M membrane and into hairless mouse skin. $^{30}$ As shown in Figure 8, a linear pattern was also observed in this study between the in vitro deposition of PPD into Strat-M membrane and its deposition into human cadaver skin. The current data again demonstrate that Strat-M could be a useful alternative to animal and human skin in permeation and deposition studies.

It is notable that the optimized NLC formulation resulted in a significantly higher in vitro deposition of PPD in both the Strat-M and human cadaver skin studies than that from the lipid solution (Figures 6 and 7). Since the in vitro deposition of PPD from the suspension was negligibly lower than that of the lipid solution and NLC formulation in the Strat-M membrane study (Figure 6), the suspension was not included in the

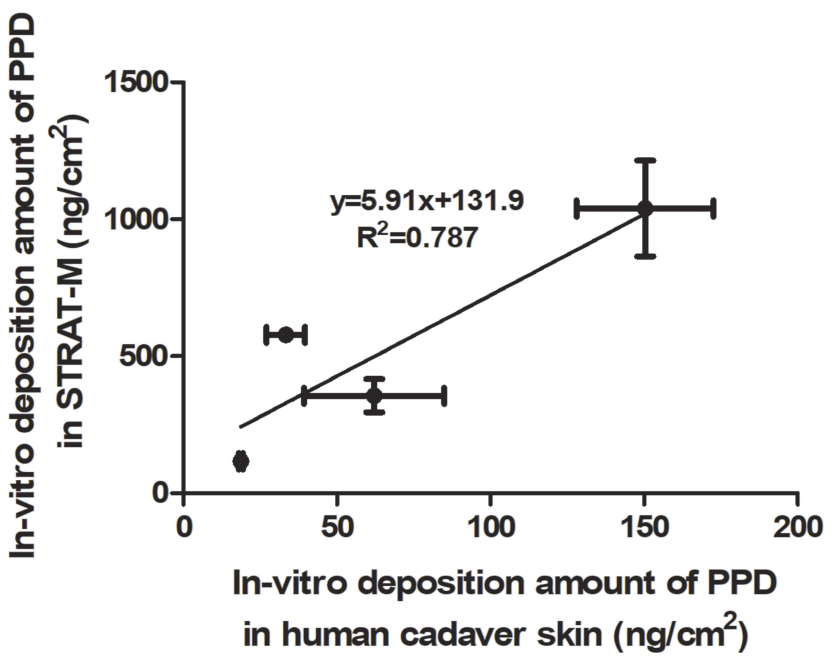

Figure 8 Correlation of the in vitro deposition amount of PPD in the Strat-M ${ }^{T M}$ membrane and the human cadaver skin at $3 \mathrm{hr}$ and $6 \mathrm{hr}$ after application of $1.0 \mathrm{~mL}$ of liquid solution or optimized NLC formulation containing $0.5 \%(w / v)$ of PPD. Data were expressed as the mean \pm standard deviation $(n=3)$. The solid line represents the fitted regression curve.

human cadaver skin deposition study (Figure 7). Based on Fick's diffusion law, the enhanced solubility of PPD in the lipid solution and NLC formulation could be a critical factor for increasing the driving force of diffusion. ${ }^{32}$ Moreover, the surfactants in the NLC formulation could further act as permeation enhancers, resulting in higher deposition of PPD than lipid solution. ${ }^{33,34} \mathrm{~A}$ higher deposited amount of PPD in the dermis layer after $6 \mathrm{hr}$ of NLC application than that of lipid solution, as seen in Figure 7B, could be explained by the effect of the surfactant in the NLC formulation. Thus, NLC formulation appears to be suitable for enhancing the skin deposition of PPD in the dermis layer, which is its target site of action. Moreover, the optimized NLC formulation with and without PPD did not show human skin irritation after a single dose application (Table 6). The excipients of the NLC formulation, which include cetyl palmitate, Miglyol 812N, Tween 20 and Poloxamer 188, are known to have good biocompatibility, and are not irritants when used for topical skin application. ${ }^{35-37}$

\section{Conclusion}

A PPD-loaded NLC formulation was successfully optimized using the Box-Behnken design. The optimized NLC formulation significantly enhanced the in vitro deposition of PPD in both Strat-M ${ }^{\mathrm{TM}}$ and human cadaver skin studies compared to that by the PPD lipid solution. Moreover, as the optimized NLC formulation did not cause any skin irritation, it could be used to enhance the topical skin deposition of poorly water-soluble ginsenosides and their deglycosylated metabolites, including PPD. 


\section{Acknowledgments}

This work was supported by Nano-Convergence Foundation funded by the Ministry of Science and ICT (MSIT, Korea) and the Ministry of Trade, Industry and Energy (MOTIE, Korea) (Project Number: R201602410).

\section{Disclosure}

Mr Min-Hwan Kim reports a patent issued to KR 101975295. Professor Dae-Duk Kim reports a patent; Korea 10-1975295. The authors report no other conflicts of interest in this work.

\section{References}

1. Miyachi Y. Photoaging from an oxidative standpoint. J Dermatol Sci. 1995;9(2):79-86.

2. Masaki H. Role of antioxidants in the skin: anti-aging effects. $J$ Dermatol Sci. 2010;58(2):85-90. doi:10.1016/j.jdermsci.2010.03.003

3. Wang CZ, Zhang Z, Wan JY, et al. Protopanaxadiol, an active ginseng metabolite, significantly enhances the effects of fluorouracil on colon cancer. Nutrients. 2015;7(2):799-814. doi:10.3390/nu7020799

4. Zhang Z, Li Z, Wu X, et al. TRAIL pathway is associated with inhibition of colon cancer by protopanaxadiol. J Pharmacol Sci. 2015;127(1):83-91. doi:10.1016/j.jphs.2014.11.003

5. Xu C, Teng J, Chen W, et al. 20(S)-protopanaxadiol, an active ginseng metabolite, exhibits strong antidepressant-like effects in animal tests. Prog Neuropsychopharmacol Biol Psychiatry. 2010;34 (8):1402-1411. doi:10.1016/j.pnpbp.2010.07.010

6. Yang Y, Lee J, Rhee MH, et al. Molecular mechanism of protopanaxadiol saponin fraction-mediated anti-inflammatory actions. $J$ Ginseng Res. 2015;39(1):61-68. doi:10.1016/j.jgr.2014.06.002

7. Han S, Lim TG, Kim JE, et al. The ginsenoside derivative 20(S)protopanaxadiol inhibits solar ultraviolet light-induced matrix metalloproteinase-1 expression. J Cell Biochem. 2017;118(11):3756-3764. doi: $10.1002 /$ jcb. 26023

8. Kirjavainen M, Urtti A, Valjakka-Koskela R, Kiesvaara J, Monkkonen J. Liposome-skin interactions and their effects on the skin permeation of drugs. Eur J Pharm Sci. 1999;7(4):279-286.

9. Maniyar MG, Kokare CR. Formulation and evaluation of spray dried liposomes of lopinavir for topical application. J Pharm Investig. 2018;49:1-12.

10. Balguri SP, Adelli GR, Majumdar S. Topical ophthalmic lipid nanoparticle formulations (SLN, NLC) of indomethacin for delivery to the posterior segment ocular tissues. Eur $J$ Pharm Biopharm. 2016;109:224-235. doi:10.1016/j.ejpb.2016.10.015

11. Beloqui A, Solinis MA, Rodriguez-Gascon A, Almeida AJ, Preat V. Nanostructured lipid carriers: promising drug delivery systems for future clinics. Nanomed-Nanotechnol. 2016;12(1):143-161. doi:10.1016/j.nano. 2015.09.004

12. Mohammadi-Samani S, Zojaji S, Entezar-Almahdi E. Piroxicam loaded solid lipid nanoparticles for topical delivery: preparation, characterization and in vitro permeation assessment. J Drug Deliv Sci Tec. 2018;47:427-433. doi:10.1016/j.jddst.2018.07.015

13. Muller RH, Radtke M, Wissing SA. Nanostructured lipid matrices for improved microencapsulation of drugs. Int J Pharm. 2002;242(12):121-128. doi:10.1016/s0378-5173(02)00180-1

14. Nnamani PO, Hansen S, Windbergs M, Lehr CM. Development of artemether-loaded nanostructured lipid carrier (NLC) formulation for topical application. Int J Pharm. 2014;477(1-2):208-217. doi:10.1016/j.ijpharm. 2014.10.004
15. Pokharkar V, Patil-Gadhe A, Kaur G. Physicochemical and pharmacokinetic evaluation of rosuvastatin loaded nanostructured lipid carriers: influence of long-and medium-chain fatty acid mixture. $J$ Pharm Invest. 2018;48:1-12.

16. Lasoń E, Sikora E, Miastkowska M, Socha P, Ogonowski J. NLC delivery systems for alpha lipoic acid: physicochemical characteristics and release study. Colloids Surf A. 2017;532:57-62. doi:10.1016/ j.colsurfa.2017.06.083

17. Muller RH, Petersen RD, Hommoss A, Pardeike J. Nanostructured lipid carriers (NLC) in cosmetic dermal products. Adv Drug Deliv Rev. 2007;59(6):522-530. doi:10.1016/j.addr.2007.04.012

18. Hao J, Fang X, Zhou Y, et al. Development and optimization of solid lipid nanoparticle formulation for ophthalmic delivery of chloramphenicol using a Box-Behnken design. Int $J$ Nanomedicine. 2011;6:683-692. doi:10.2147/IJN.S17386

19. Ravi PR, Aditya N, Kathuria H, Malekar S, Vats R. Lipid nanoparticles for oral delivery of raloxifene: optimization, stability, in vivo evaluation and uptake mechanism. Eur J Pharm Biopharm. 2014;87 (1):114-124. doi:10.1016/j.ejpb.2013.12.015

20. Abdelbary AA, AbouGhaly MH. Design and optimization of topical methotrexate loaded niosomes for enhanced management of psoriasis: application of Box-Behnken design, in-vitro evaluation and invivo skin deposition study. Int $J$ Pharm. 2015;485(1-2):235-243. doi:10.1016/j.ijpharm.2015.03.020

21. Ranch KM, Maulvi FA, Naik MJ, Koli AR, Parikh RK, Shah DO. Optimization of a novel in situ gel for sustained ocular drug delivery using Box-Behnken design: in vitro, ex vivo, in vivo and human studies. Int J Pharm. 2019;554:264-275. doi:10.1016/j.ijpharm.2018.11.016

22. Salunkhe SS, Bhatia NM, Pokharkar VB, Thorat JD, Bhatia MS. Topical delivery of Idebenone using nanostructured lipid carriers: evaluations of sun-protection and anti-oxidant effects. $J$ Pharm Investig. 2013;43(4):287-303. doi:10.1007/s40005-0130079-y

23. Lee SE, Lee JK, Jang WS, et al. Enhancement of stability and controlled drug release of lipid nanoparticles by modified solventevaporation method. Colloid Surf A. 2016;508:294-300. doi:10.1016/ j.colsurfa.2016.08.043

24. Kim KT, Kim MH, Park JH, et al. Microemulsion-based hydrogels for enhancing epidermal/dermal deposition of topically administered 20(S)-protopanaxadiol: in vitro and in vivo evaluation studies. J Ginseng Res. 2018;42(4):512-523. doi:10.1016/j. jgr.2017.07.005

25. Jee JP, Pangeni R, Jha SK, Byun Y, Park JW. Preparation and in vivo evaluation of a topical hydrogel system incorporating highly skinpermeable growth factors, quercetin, and oxygen carriers for enhanced diabetic wound-healing therapy. Int $J$ Nanomedicine. 2019;14:5449-5475. doi:10.2147/IJN.S213883

26. Tummala S, Gowthamarajan K, Satish Kumar MN, et al. Formulation and optimization of oxaliplatin immuno-nanoparticles using Box-Behnken design and cytotoxicity assessment for synergistic and receptor-mediated targeting in the treatment of colorectal cancer. Artif Cells Nanomed Biotechnol. 2016;44(8):1835-1850. doi:10.3109/21691401.2015.1111226

27. Han F, Li SM, Yin R, Liu HZ, Xu L. Effect of surfactants on the formation and characterization of a new type of colloidal drug delivery system: nanostructured lipid carriers. Colloid Surf A. 2008;315(13):210-216. doi:10.1016/j.colsurfa.2007.08.005

28. Ferreira M, Chaves LL, Lima SA, Reis S. Optimization of nanostructured lipid carriers loaded with methotrexate: a tool for inflammatory and cancer therapy. Int $J$ Pharm. 2015;492(1-2):65-72. doi:10.1016/j.ijpharm.2015.07.013

29. Teeranachaideekul V, Souto EB, Junyaprasert VB, Muller RH. Cetyl palmitate-based NLC for topical delivery of Coenzyme Q(10) development, physicochemical characterization and in vitro release studies. Eur J Pharm Biopharm. 2007;67(1):141-148. doi:10.1016/j. ejpb.2007.01.015 
30. Uchida T, Kadhum WR, Kanai S, Todo H, Oshizaka T, Sugibayashi K. Prediction of skin permeation by chemical compounds using the artificial membrane, Strat-M. Eur J Pharm Sci. 2015;67:113-118. doi:10.1016/j.ejps.2014.11.002

31. Yun YE, Jung YJ, Choi YJ, Choi JS, Cho YW. Artificial skin models for animal-free testing. $J$ Pharm Investig. 2018;48(2):215-223. doi:10.1007/s40005-018-0389-1

32. Moser K, Kriwet K, Naik A, Kalia YN, Guy RH. Passive skin penetration enhancement and its quantification in vitro. Eur $J$ Pharm Biopharm. 2001;52(2):103-112.

33. Hajjar B, Zier K-I, Khalid N, Azarmi S, Löbenberg R. Evaluation of a microemulsion-based gel formulation for topical drug delivery of diclofenac sodium. $J$ Pharm Investig. 2018;48(3):351-362. doi:10.1007/s40005-017-0327-7
34. Mbah CC, Builders PF, Agubata CO, Attama AA. Development of ethosomal vesicular carrier for topical application of griseofulvin: effect of ethanol concentration. $J$ Pharm Investig. 2019;49(1):2736. doi:10.1007/s40005-017-0367-z

35. Morales JO, Valdes K, Morales J, Oyarzun-Ampuero F. Lipid nanoparticles for the topical delivery of retinoids and derivatives. Nanomedicine-Uk. 2015;10(2):253-269. doi:10.2217/nnm.14.159

36. Khurana S, Bedi PMS, Jain NK. Preparation and evaluation of solid lipid nanoparticles based nanogel for dermal delivery of meloxicam. Chem Phys Lipids. 2013;175:65-72. doi:10.1016/j.chemphyslip.2013.07.010

37. MagalhãesI MSF, FechineII FV, de MacedoIII RN, et al. Effect of a combination of medium chain triglycerides, linoleic acid, soy lecithin and vitamins $\mathrm{A}$ and $\mathrm{E}$ on wound healing in rats1. Acta Cirúrgica Brasileira. 2008;23:3.

\section{Publish your work in this journal}

The International Journal of Nanomedicine is an international, peerreviewed journal focusing on the application of nanotechnology in diagnostics, therapeutics, and drug delivery systems throughout the biomedical field. This journal is indexed on PubMed Central, MedLine, CAS, SciSearch ${ }^{\mathbb{R}}$, Current Contents ${ }^{\mathbb{R}} /$ Clinical Medicine, $^{2}$
Journal Citation Reports/Science Edition, EMBase, Scopus and the Elsevier Bibliographic databases. The manuscript management system is completely online and includes a very quick and fair peer-review system, which is all easy to use. Visit http://www.dovepress.com/ testimonials.php to read real quotes from published authors. 